\title{
Validity and reliability of the Turkish version of the adult ADHD Self-Re- port Screening Scale for DSM-5
}

\author{
Herdem Aslan Genç ${ }^{1}$ (D), Neşe Yorguner ${ }^{2}$ (D), Serkut Bulut ${ }^{2}$ (D), Gresa Carkaxhiu Bulut ${ }^{3}$ (D), Ömer Aydemir ${ }^{4}$ (D), \\ Yankı Yazgan ${ }^{5,6}$
}

\author{
${ }^{1}$ Department of Child Psychiatry, Koç University Hospital, İstanbul, Turkey \\ ${ }^{2}$ Department of Psychiatry, Marmara University Pendik Training and Research Hospital, İstanbul, Turkey \\ ${ }^{3}$ Department of Child and Adolescent Psychiatry, Maltepe University School of Medicine, İstanbul, Turkey \\ ${ }^{4}$ Department of Psychiatry, Celal Bayar University School of Medicine, Manisa, Turkey \\ ${ }^{5}$ Department of Child Psychiatry, Marmara University School of Medicine, İstanbul, Turkey \\ ${ }^{6}$ Child Study Center, Yale University School of Medicine, New Haven, CT, USA
}

\begin{abstract}
Background: Approximately half of the children with attention-deficit hyperactivity disorder continue to meet diagnostic criteria in adulthood. The prevalence of adult attention-deficit hyperactivity disorder is reported between $2.5 \%$ and $4.4 \%$ and is associated with significant impairment in quality of life and increased psychiatric comorbidity. Attention-deficit hyperactivity disorder in adults remains mostly undiagnosed and/or untreated despite the availability of effective treatments. The majority of people who do not receive necessary treatment are in the nonclinical or nonpsychiatric clinical population. Screening is an important step for diagnosing adults with attention-deficit hyperactivity disorder. Yet, there are no valid and reliable screening questionnaires calibrated for the Diagnostic and Statistical Manual for Mental Disorders-5 in Turkish.
\end{abstract}

Aims: We aimed to test the reliability and the validity of the Adult Attention-Deficit Hyperactivity Disorder Self-Report Scale-5 screening questionnaire designed according to DSM-5 in the Turkish population.

Study Design: Methodological and cross-sectional study.

Methods: The translation was carried out according to the World Health Organization Composite International Diagnostic Interview translation guide using a linguistic adaptation approach. We used a convenience sampling method to recruit an individual with adult attention-deficit hyperactivity disorder $(n=68)$ and a control group $(n=68)$. The participants completed a sociodemographic form, 6-items Adult Attention-Deficit Hyperactivity Disorder Self-Report Scale-5, and the previous version 18-items Adult Attention-Deficit Hyperactivity Disorder Self-Report Scale-v1.1 for the concurrent validity analysis. For the diagnostic validity, clinical diagnosis made by psychiatrists according to the Diagnostic and Statistical Manual for Mental Disorders- 5 criteria was used. Internal consistency and item-total correlation coefficients, exploratory factor analyses, correlation with Adult Attention-Deficit Hyperactivity Disorder Self-Report Scale-v1.1, and receiver operating characteristic curve analysis were conducted.

Results: The internal consistency measured by Cronbach alpha was 0.869. Item-total correlation coefficients were calculated to be between 0.602 and 0.717 , and the correlations were statistically significant $(P<0.0001)$. The Adult Attention-Deficit Hyperactivity Disorder Self-Report Scale-5 showed to have a unidimensional factor structure explaining $60.54 \%$ of the variance. The correlation between Adult Attention-Deficit Hyperactivity Disorder Self-Report Scale-5 and Adult Attention-Deficit Hyperactivity Disorder Self-Report Scale-v1.1 total score was calculated as $0.992(P<0.0001)$, and that between Adult Attention-Deficit Hyperactivity Disorder Self-Report Scale-5 and Adult Attention-Deficit Hyperactivity Disorder Self-Report Scale-v1.1 attention-deficit subdimension was $0.868(P<0.0001)$. In the receiver operating characteristic analysis of Adult Attention-Deficit Hyperactivity Disorder Self-Report Scale-5, the area under the curve was found to be 0.916 . The cut-off score was calculated as 9 of 10 with a sensitivity of $85.2 \%$ and specificity of $89.7 \%$.

Conclusion: Adult Attention-Deficit Hyperactivity Disorder Self-Report Scale-5 is a valid and reliable self-report measure to assess and screen attention-deficit hyperactivity disorder in the Turkish population. It may be useful for both clinical and population studies.

Address for Correspondence: Herdem Aslan Genç, Department of Child Psychiatry, Koç University Hospital, İstanbul, Turkey

e-mail: hgenc@kuh.ku.edu.tr

Received: May 14, 2020 Accepted: September 29, 2020 •DOI: 10.4274/balkanmedj.galenos.2020.2020.5.119

Available at www.balkanmedicaljournal.org

ORCID iDs of the authors: H.A.G. 0000-0002-1130-999X; N.Y. 0000-0001-8411-0466; S.B. 0000-0003-3044-3999; G.C.B. 0000-0002-0582-4936; Ö.A. 0000-0003-3050-1263.

Cite this article as:

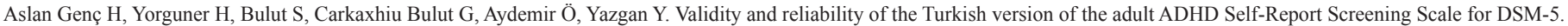
Balkan Med J. 2021;38(2):111-115.

Copyright@Author(s) - Available online at http://balkanmedicaljournal.org/ 


\section{INTRODUCTION}

Attention-deficit hyperactivity disorder (ADHD) is the most common neurodevelopmental disorder in childhood characterized by inattentiveness, hyperactivity, and impulsivity. ADHD was, until recently, perceived as a childhood disorder with symptoms typically remitting by adulthood. ${ }^{1,2}$ The worldwide prevalence of ADHD in childhood is around $5.9 \%$, whereas the estimated adult prevalence is reported to range from 2.5 to $4.4 \%$ in recent reviews. ${ }^{3,4}$ Approximately $40-60 \%$ of children with ADHD continue to meet the criteria for the disorder even when they become adults and present with significant impairment in academic, occupational, and social functioning as well as high psychiatric comorbidity. ${ }^{5}$ The most common comorbid psychiatric disorders in adult ADHD are reported to be mood disorders, anxiety disorders, and substance use disorders. ${ }^{3,4}$ Adults with ADHD are more likely to suffer from occupational difficulties, unemployment, risky behaviors, criminality (especially in the presence of comorbid antisocial personality disorder), interpersonal problems, alcohol and substance misuse, motor vehicle accidents, increased healthcare expenses, and premature death. ${ }^{6,7}$ Mortality rates have been shown to be higher in people with adult ADHD, with accidents being the most common leading cause. ${ }^{8}$ Being associated with significant impairment in quality of life, increased psychiatric comorbidity, and disability rates, ADHD in adults remains mostly undiagnosed and/or untreated despite the availability of effective treatments., 3 -11 The majority of people who do not receive necessary treatment are in the nonclinical or nonpsychiatric clinical population. The burden of adult ADHD is further increased by the limited access to treatment, which largely results from underdiagnosis.

The diagnosis of adult ADHD is made through a comprehensive psychiatric assessment, which involves a thorough identification of symptoms and behavioral problems consistent with Diagnostic and Statistical Manual of Mental Disorders (DSM)-5 criteria, as well as the evaluation of the level of impairment by ruling out other psychiatric disorders that may contribute to the symptoms. Before implementing such comprehensive assessments, brief and efficient screeners that can be used in both clinical and nonclinical populations for adult ADHD are needed in terms of feasibility. Therefore, the use of solid screening instruments appears to be as crucial as the implementation of more elaborate diagnostic procedures.

Among the valid screening scales that are currently in use, most are calibrated to DSM-IV criteria. However, with DSM-5, the required number of symptoms has been reduced from 6 to 5 and the age of onset has been increased from 7 to 12 years. The Adult ADHD Self-Report Scale (ASRS)-v1.1 is a valid tool assessing adult ADHD symptoms and has recently been updated according to DSM-5. The DSM-5 ASRS screening scale (ASRS-5) has been tested in both clinical and population samples and has shown great concordance with blinded clinical diagnostic interviews. With its 6 items derived from a pool of 29 items, using a machine learning algorithm, the ASRS-5 has 91.4\% sensitivity and 96\% specificity in the population setting and $91.9 \%$ sensitivity and $74 \%$ specificity in the clinical sample. ${ }^{12}$ The adult ADHD prevalence in Turkey is currently unknown, which points out the need for reliable and val- id screening questionnaires that are developed on the basis of the DSM-5 criteria and may operate in both population and clinical samples. In this study, we aimed to test the reliability and validity of the ASRS-5 questionnaire in the Turkish population.

\section{MATERIAL AND METHODS}

For the adaptation of ASRS-5 into Turkish, written permission has been obtained from the developers of the original scale. The translation has been carried out according to the World Health Organization (WHO) Composite International Diagnostic Interview translation guide. The translation process involved the following steps: parallel translations, expert panel review, pretesting and cognitive interviewing, final version, and documentation. The initial translations were separately made by psychiatrists who had at least 2 years of clinical experience. These separate versions were then reviewed and finalized by the expert panel, which comprised senior psychiatrists working in the field. In the cognitive interviewing step of the adaptation process, the final revised version of the scale was applied to a heterogeneous group of 10 people of various ages, both sex, and educational levels; each interview was then reported. After the approval of the translation report containing the requested information about the process by the corresponding author/developer, the Turkish version of the scale was published at https:// www.hcp.med.harvard.edu/ncs/asrs.php.

A nonprobability, convenience sampling method was used. A power analysis using the mean values of the same scale from a previous study ${ }^{18}$ revealed a total sample size of 40 (20 participants for each group) with a power of 0.80 , although we have recruited every possible participant in the duration of the study. Patients who applied consecutively to the psychiatry outpatient clinics of the 2 university hospitals and received an ADHD diagnosis according to DSM-5 criteria $(n=68)$ were assigned to the ADHD group. The control group $(n=68)$ consisted of participants who applied to the outpatient units of the 2 university hospitals other than psychiatry and did not have a previous diagnosis of ADHD, as also confirmed by a DSM-5-based diagnostic interview. The recruitment of the control group was conducted using a snowball sampling method, and the participants were asked to inform others about participating in the study. The inclusion criteria were being aged between 18 and 44 years and having enough literacy to fill in the study questionnaires. Individuals with chronic physical and neurological diseases, intellectual disability, or a diagnosis of genetic syndrome were excluded.

\section{Structured clinical interview for DSM-5 disorders}

Structured Clinical Interview for DSM-5 (SCID-5) is a semistructured clinical interview for making DSM-5 diagnoses. SCID-5 and earlier versions have been used frequently to make diagnoses and select samples for research in the literature and have been utilized as a gold-standard instrument to assess psychiatric disorders in clinical research. ${ }^{13,14}$ The validity and reliability study of the Turkish version of SCID-5 has been performed by Elbir et al. ${ }^{15}$ The authors were trained for the application of the SCID-5 and held a preliminary meeting before the interviews were initiated. In this study, the ADHD module of SCID-5 was used. The interrater reliability analysis of the different versions of the SCID-5 ADHD 
module revealed kappa coefficient values ranging between 0.80 and 1.00, suggesting excellent interrater reliability. ${ }^{15,16}$

\section{DSM-5 ASRS Screening Scale}

ASRS-5 is an ADHD screening scale developed by the WHO in line with DSM-5 diagnostic criteria. It is a 5-point Likert-type scale consisting of 6 items. The validity study of the ASRS- 5 has been conducted by Üstün et al., and it revealed excellent psychometric properties. The sensitivity of the original form of ASRS-5 was $91.4 \%$, the specificity was $96 \%$, and the value of the area under the curve (AUC) was 0.94. It has been demonstrated that it is correlated with clinical diagnoses in both population and clinical samples. ${ }^{12}$

\section{Adult ADHD Self-Report Scale}

ASRS-v1.1 is an 18-item scale that questions ADHD symptoms on the basis of DSM-IV criteria in adults. It is a 5-point Likert scale. The first 9 items of the scale question the symptoms of attention deficit, and the next 9 items question the symptoms of hyperactivity and impulsivity. In the Turkish validity and reliability study of ASRS-v1.1 in 2009, the Turkish form of ASRS has been shown to be a valid and reliable tool for screening adult ADHD symptoms. ${ }^{17}$

\section{Statistical analyses}

Data analyses were conducted using the Statistical Package for the Social Sciences, version 26 (IBM SPSS Corp.; Armonk, NY, USA). Kolmogorov-Smirnov tests were used to explore the normality distribution of the parametric data. Independent samples $t$-test for parametric values and Chi-square test for categorical variables were applied to compare sociodemographic variables, such as age, sex, and educational level. Cronbach's alpha internal consistency coefficient and item-total score correlation coefficients were calculated for reliability analyses. To test the construct validity, principal factor analyses were conducted. The factors with an eigenvalue $>1$ were assessed, and the items with a factor loading $>0.4$ were taken into consideration in the statistical analyses. For criterion validity, a receiver operating characteristic (ROC) analysis was performed by calculating the specificity and sensitivity between those who received a diagnosis using SCID-5 and those who did not. Pearson correlation analysis was performed to assess the simultaneous validity of ASRS-5 with ASRS-v1.1. A probability level of $P \leq 0.05$ was used to indicate statistical significance.

The local ethics committee of the medical faculty approved the study, which was conducted in accordance with the ethical standards set out in the 1964 Declaration of Helsinki and the relevant regulations of the United States Health Insurance Portability and Accountability Act. Informed written consent was obtained from all individuals who volunteered to participate in the study.

\section{RESULTS}

The sociodemographic properties, such as sex, age, socioeconomic status, and education level, were similar between the groups (Table 1).

The internal consistency measured by Cronbach's alpha was 0.877 . Item-total correlation coefficients were calculated to be between 0.616 and 0.728 , and the correlations were statistically significant $(P<0.0001)$ (Table 2).
The ASRS-5 showed to have a unidimensional factor structure, and the eigenvalue was calculated to be 3.632 . The unidimensional factor structure explained $60.54 \%$ of the variance. The factor loadings of the items ranged between 0.721 and 0.816 (Table 2).

The correlation between the ASRS-5 and the total and the subdimensions of ASRS-v1.1 were examined. The correlation between ASRS-5 and ASRS-v1.1 total score was calculated to be 0.935 ( $P$ $<0.0001)$, the correlation between ASRS-5 and ASRS-v1.1 attention-deficit subdimension was $0.887(P<0.0001)$, and the correlation between ASRS-5 and ASRS-v1.1 hyperactivity/impulsivity subdimension was $0.893(P<0.0001)$.

To examine the discrimination between patients assessed with ASRS-5 and healthy volunteer groups, the total scores obtained by the groups were compared. The score obtained by the ADHD group $(14.37 \pm 4.30)$ was higher than the score obtained by the healthy control group $(5.89 \pm 3.57)(\mathrm{F}=-12.321, P<0.0001)$ (Table 3$)$.

In the ROC analysis of ASRS-5, which covered the ADHD group and the control group, the AUC was found to be 0.913 (Figure 1). The specificity-sensitivity analysis of the scale was calculated according to the ROC analyses, and it revealed the cut-off score of 10 , which means that scores $<10$ indicates the absence of an

TABLE 1. Comparison of the sociodemographic variables of the participants

\begin{tabular}{lccc}
\hline Variables & ADHD group & Control group & $P$ \\
\hline Age, mean \pm SD & $29.22 \pm 8.80$ & $31.11 \pm 6.13$ & 0.155 \\
Sex, n (\%) & & & \\
Female & $37(54.4)$ & $37(54.4)$ & 1.000 \\
Male & $31(45.6)$ & $31(45.6)$ & \\
Socioeconomic status, n (\%) & & & \\
Low & $9(13.2)$ & $6(8.8)$ & 0.819 \\
Moderate & $46(67.6)$ & $48(70.6)$ & \\
High & $13(19.1)$ & $14(20.6)$ & \\
Educational level, n (\%) & & & \\
Elementary & $2(2.9)$ & $9(13.2)$ & 0.150 \\
High school & $19(27.9)$ & $15(22.1)$ & \\
University & $47(69.1)$ & $44(64.7)$ & \\
\hline
\end{tabular}

Analysis was conducted using Pearson Chi-square tests.

ADHD, attention-deficit hyperactivity disorder; SD, standard deviation.

TABLE 2. Item-total correlation coefficients and factor loadings of the ASRS5

\begin{tabular}{lcc}
\hline Item & Item-total correlation coefficients & Component \\
\hline Item 1 & 0.678 & 0.784 \\
Item 2 & 0.616 & 0.731 \\
Item 3 & 0.644 & 0.755 \\
Item 4 & 0.725 & 0.820 \\
Item 5 & 0.728 & 0.826 \\
Item 6 & 0.702 & 0.803 \\
\hline ADHD, attention-deficit hyperactivity disorder; ASRS, Adult ADHD Self-Report Scale.
\end{tabular}


TABLE 3. Comparison of ASRS-v1.1 and ASRS-5 Mean Scores Between Groups

\begin{tabular}{lccc}
\hline & $\begin{array}{c}\text { ADHD group } \\
(\mathrm{n}=68) \\
\text { Mean } \pm \mathrm{SD}\end{array}$ & $\begin{array}{c}\text { Control group } \\
(\mathrm{n}=68) \\
\text { Mean } \pm \mathrm{SD}\end{array}$ & $P$ \\
\hline ASRS version & $23.53 \pm 6.64$ & $9.97 \pm 4.94$ & $<0.0001$ \\
\hline ASRS-v1.1 IA subdimension & $21.01 \pm 6.48$ & $8.36 \pm 6.18$ & $<0.0001$ \\
ASRS-v1.1 HI subdimension & $44.54 \pm 11.74$ & $18.33 \pm 9.99$ & $<0.0001$ \\
ASRS-v1.1 total & $14.37 \pm 4.30$ & $5.89 \pm 3.57$ & $<0.0001$ \\
\hline
\end{tabular}

ADHD, attention-deficit hyperactivity disorder; ASRS, Adult ADHD Self-Report Scale; IA, inattention; HI, hyperactivity.

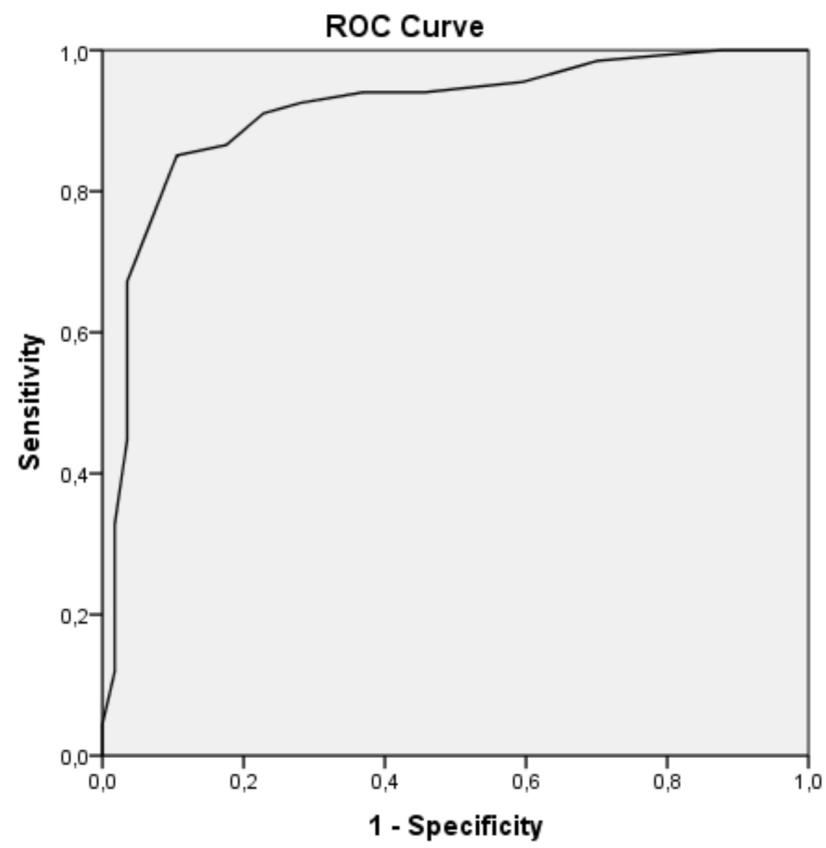

FIG. 1. ROC curve of the ASRS-5 total scores and sensitivity-specificity analyses of the ASRS-5 according to the ROC analysis.

ADHD, attention-deficit hyperactivity disorder; ASRS, Adult ADHD Self-Report Scale; ROC, receiver operating characteristic.

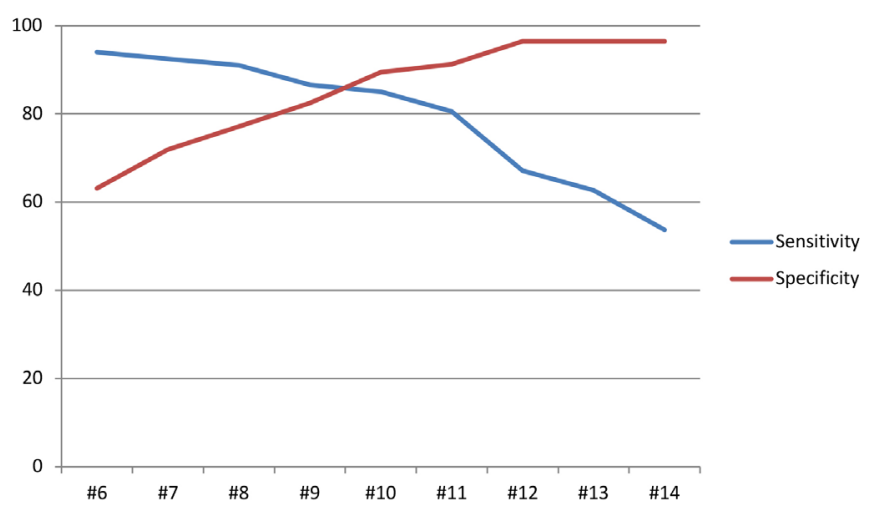

FIG. 2. Determination of optimal cut-off point according to the receiver operating characteristic analysis of the ASRS-5.

ADHD, attention-deficit hyperactivity disorder; ASRS, Adult ADHD Self-Report Scale. $X$ axis, ASRS5 total score; $Y$ axis: Percentage (\%)
ADHD diagnosis, and scores $\geq 10$ indicate a probable ADHD diagnosis (Figure 2). The sensitivity of this cut-off score was calculated as $85.1 \%$, and the specificity was calculated as $89.5 \%$. The positive predictive value was $90.5 \%$, and the negative predictive value was $83.6 \%$.

\section{DISCUSSION}

Despite the well-recognized overall burden associated with the disease and the availability of effective treatments, ADHD remains often underdiagnosed and undertreated during adulthood. ${ }^{18}$ To overcome this shortcoming, the use of practical screening tools appropriate for both clinical and nonclinical settings is crucial. Existing self-report scales for ADHD in the Turkish language are problematic in this regard given that ${ }^{1}$ they lack screener versions, which limits their widespread clinical use, and ${ }^{2}$ none of them are based on DSM-5 criteria. With the need for a brief, effective, and DSM-5-based screening tool being considered, this study was set out with the aim of assessing the reliability and validity of the Turkish version of the ASRS-5, which has proven to be a valuable tool to screen ADHD in adults with excellent psychometric properties. ${ }^{12}$ With respect to the research question, we investigated the association between the ASRS-5 score with clinical diagnoses and the ASRS-v1.1 score. The results of our reliability and validity study of the Turkish version of the ASRS-5 indicate that ASRS-5 is a valid and reliable tool that can be used in the Turkish population to screen ADHD in adults.

\section{Reliability analyses}

Both the internal consistency (Cronbach's alpha $=0.87$ ) and the item-total correlation coefficients (between 0.721 and 0.816 ) were found to be high. The internal consistency values of other ADHD symptom rating scales are 0.88 and 0.93 for ASRS-v1.1 and Wender Utah Rating Scale (WURS), respectively. ${ }^{17,19}$ The item-total correlation coefficients of ASRS-5 were found to range from 0.72 to 0.82 , which indicates an increased reliability and homogeneity compared with that of other relevant scales. The same range for ASRS-v1.1 was not analyzed, and for WURS, it was 0.31-0.75. According to our findings, ASRS-5 has satisfactory reliability values.

\section{Validity analyses}

The factor analyses to investigate the construct validity of the ASRS-5 revealed a single factor, and items have been shown to represent a single structure. According to the single-factor structure, no further Varimax rotation was conducted. This one-factor structure explains $60.4 \%$ of the variance. The factor analyses of the old version of the ASRS screener also revealed a single-factor structure. ${ }^{20,21}$

To demonstrate the content validity of the ASRS-5, we ran correlation analyses with ASRS-v1.1. The correlation coefficients were found to be between 0.868 and 0.929 , which represent a very strong correlation between the 18-item full-scale and the 6-item screener. The comparison analyses, which aimed to show the differentiation capacity of the ASRS-5 between patients with ADHD and healthy individuals showed that people with ADHD have significantly higher scores than those with ADHD and that ASRS-5 is adequate to 
differentiate that. We also conducted ROC analyses to investigate the content validity and found an AUC value $>0.9$. Whereas an AUC value $>0.7$ is deemed acceptable for many scales, a value $>0.9$ is considered outstanding. Our finding is comparable with that of the original version of the ASRS-5, where the AUC value was found to be 0.94 . The AUC value of the previous screener was ranging between 0.75 and 0.85 , which also points out the necessity and the superiority of the updated version. ${ }^{21,22}$ The results of our validity analyses are comparable with those of the original study, where the specificity and sensitivity values were $96 \%$ and $91.4 \%$, respectively. Taken together, the Turkish version of the ASRS- 5 is suggested to be a valid and reliable tool to assess and screen ADHD.

The study has several limitations. First, performing a detailed and time-taking diagnostic interview with all participants may have resulted in a relatively small sample size, which nevertheless allowed to properly perform the preplanned statistical analyses. Second, we did not make language validity analyses, which would have affected the validity and reliability of the scale positively. Finally, the study was conducted in a clinical sample and did not include a population sample, and further research in a community-based sample would be needed for establishing the use of ASRS for screening purposes; still, the original scale has been reported to have similar psychometric properties in the general population and clinical samples. ${ }^{12}$

Turkish version of the ASRS-5 stands out as a valid, reliable, and promising tool to assess and screen ADHD in the Turkish population. Considering its effectiveness and practicality, it may be useful for both clinical and population studies.

Ethics Committee Approval: Ethics committee approval was received for this study from the Ethics Committee of Sakarya University Medical Faculty with the decision number of $71522473 / 050.01 .04 / 95$.

Patient Consent for Publication: Written informed consent was obtained from all individuals who volunteered to participate in the study.

Data-sharing Statement: N/A.

Acknowledgments: The authors would like to thank Prof. Ronald C. Kessler for providing approval for the translation of the ASRS-5 and Teresa Gorman for the assistance during the translation process.

Author Contributions: Concept - H.A.G., G.C.B., S.B., N.Y., Y.Y.; Design - H.A.G., G.C.B., S.B., N.Y., Ö.A.; Supervision - Y.Y., Ö.A.; Resources - H.A.G., G.C.B., S.B., N.Y., Y.Y.; Materials - BG.C.B., S.B., N.Y.; Data Collection and/or Processing - G.C.B., S.B., N.Y., H.A.G.; Analysis and/or Interpretation - Ö.A., H.A.G., G.C.B., N.Y.; Literature Review - H.A.G., S.B., N.Y., G.C.B.; Writing - H.A.G., S.B., N.Y., G.C.B., Y.Y., Ö.A.; Critical Review - Y.Y., Ö.A., S.B., H.A.G., N.Y., G.C.B.

Conflict of Interest: The authors have no conflicts of interest to declare.

Funding: The authors declared that this study had received no financial support.

\section{REFERENCES}

1. Hill JC, Schoener EP. Age-dependent decline of attention deficit hyperactivity disorder. Am J Psychiatry. 1996;153(9):1143-1146. [Crossref]

2. Shaffer D. Attention deficit hyperactivity disorder in adults. Am J Psychiatry. 1994;151(5):633-638. [Crossref]
3. Fayyad J, De Graaf R, Kessler R, et al. Cross-national prevalence and correlates of adult attention-deficit hyperactivity disorder. Br J Psychiatry. 2007;190:402-409. [Crossref]

4. Kessler RC, Adler L, Barkley R, et al. The prevalence and correlates of adult ADHD in the United States: results from the National Comorbidity Survey Replication. Am J Psychiatry. 2006;163(4):716-723. [Crossref]

5. Barkley RA, Fischer M, Smallish L, Fletcher K. The persistence of attention-deficit/ hyperactivity disorder into young adulthood as a function of reporting source and definition of disorder. J Abnorm Psychol. 2002;111(2):279-289. [Crossref]

6. Biederman J, Faraone SV, Spencer TJ, Mick E, Monuteaux MC, Aleardi M. Functional impairments in adults with self-reports of diagnosed ADHD: A controlled study of 1001 adults in the community. J Clin Psychiatry. 2006;67(4):524-540. [Crossref]

7. Faraone SV, Silverstein MJ, Antshel K, et al. The Adult ADHD Quality Measures Initiative. J Atten Disord. 2019;23(10):1063-1078. [Crossref]

8. Dalsgaard S, Østergaard SD, Leckman JF, Mortensen PB, Pedersen MG. Mortality in children, adolescents, and adults with attention deficit hyperactivity disorder: a nationwide cohort study. Lancet. 2015;385:2190-2196. [Crossref]

9. Newcorn JH, Weiss M, Stein MA. The complexity of ADHD: diagnosis and treatment of the adult patient with comorbidities. CNS Spectr. 2007;12:1-14 [Crossref]

10. Retz W, Retz-Junginger P, Thome J, Rösler M. Pharmacological treatment of adult ADHD in Europe. World J Biol Psychiatry. 2011;12(Suppl 1):89-94. [Crossref]

11. Surman CBH, Hammerness PG, Pion K, Faraone SV. Do stimulants improve functioning in adults with ADHD? A review of the literature. Eur Neuropsychopharmacol. 2013;23(6):528-533. [Crossref]

12. Ustun B, Adler LA, Rudin C, et al. The World Health Organization Adult Attention-Deficit/Hyperactivity Disorder Self-Report Screening Scale for DSM-5. JAMA Psychiatry. 2017;74(5):520-527. [Crossref]

13. Rogers R. Handbook of Diagnostic and Structured Interviewing. New York, NY: Guilford Press; 2001.

14. APA Work Group on Psychiatric Evaluation. Practice Guideline for Psychiatric Evaluation of Adults. Arlington, VA: American Psychiatric Association; 2016.

15. Elbir M, Alp Topbaș Ö, Bayad S, et al. Adaptation and Reliability of the Structured Clinical Interview for DSM-5-Disorders - Clinician Version (SCID-5/CV) to the Turkish Language. Turk Psikiyatri Derg. 2019;30(1):51-56. [Crossref]

16. Osório FL, Loureiro SR, Hallak JEC, et al. Clinical validity and intrarater and test-retest reliability of the Structured Clinical Interview for DSM-5-Clinician Version (SCID-5-CV). Psychiatry Clin Neurosci. 2019;73(12):754-760. [Crossref]

17. Dogan S, Öncü B, Varol-Saraçoglu G, Küçükgöncü S. Validity and reliability of the Turkish version of the Adult ADHD Self-Report Scale (ASRS-v1. 1). Anadolu Psikiyatri Derg. 2009;10:77-87.

18. Fayyad J, Sampson NA, Hwang I, et al. The descriptive epidemiology of DSM-IV Adult ADHD in the World Health Organization World Mental Health Surveys. Atten Defic Hyperact Disord. 2017;9(1):47-65. [Crossref]

19. Oncu B, Olmez S, Senturk V. Validity and reliability of the Turkish version of the Wender Utah Rating Scale for attention-deficit/hyperactivity disorder in adults. Turk Psikiyatri Derg 2005;16:252-9.

20. Sanchez-Garcia M, Fernandez-Calderon F, Carmona-Marquez J, Chico-Garcia M, Velez-Moreno A, Perez-Gomez L. Psychometric properties and adaptation of the ASRS in a Spanish sample of patients with substance use disorders: Application of two IRT Rasch models. Psychol Assess. 2015;27(2):524-533. [Crossref]

21. Somma A, Borroni S, Fossati A. Construct validity and diagnostic accuracy of the Italian translation of the 18-Item World Health Organization Adult ADHD Self-Report Scale (ASRS-18) Italian translation in a sample of community-dwelling adolescents. Psychiatry Res. 2019;273:753-758. [Crossref]

22. Takeda T, Tsuji Y, Kurita H. Psychometric properties of the Japanese version of the Adult Attention-deficit hyperactivity disorder (ADHD) Self-Report Scale (ASRS-J) and its short scale in accordance with DSM-5 diagnostic criteria. Res Dev Disabil. 2017;63:59-66. [Crossref] 